\title{
Opinión del jefe inmediato sobre el desempeño de los egresados de la Licenciatura en Enfermería'
}

\author{
Silvia Crespo Knopfler² \\ Adiel Agama Sarabia ${ }^{3}$ \\ María Susana González Velázquez ${ }^{4}$ \\ Belinda de la Peña León 5 \\ Marisol Torres Medina'
}

doi:10.11144/Javeriana.ie18-2.ojid

Como citar: Crespo Knopfler S, Agama Sarabia A, González Velázquez MS, De la Peña León B, Torres Medina M. Opinión del jefe inmediato sobre el desempeño de los egresados de la Licenciatura en Enfermería. Investig Enferm. Imagen Desarr. 2016;18(2):107-120. http://dx.doi.org/10.11144/Javeriana.ie18-2.ojid

1. Artículo original de investigación. Fecha de recepción: 11 de mayo de 2015. Fecha de aceptación: 4 de diciembre de 2015.

2. Doctora en Educación. Profesora de tiempo completo titular B, Carrera de Enfermería, Facultad de Estudios Superiores Zaragoza, Universidad Autónoma de México. Correo electrónico: crespo.knopfer@hotmail.com

3. Maestro en Enfermeria. Profesor de tiempo completo asociado B de la Carrera de Enfermería, Facultad de Estudios Superiores Zaragoza, Universidad Autónoma de México. Correo electrónico: agama.adiel@gmail.com

4. Doctora en Educación. Profesora de tiempo completo titular B de la Carrera de Enfermería, Facultad de Estudios Superiores Zaragoza, Universidad Autónoma de México. Correo electrónico: susanagove@msn.com

5. Maestra en Educación. Técnica académica titular A de la Carrera de Enfermería, Facultad de Estudios Superiores Zaragoza, Universidad Autónoma de México. Correo electrónico: beli7207@yahoo.es

6. Pasante de la Licenciatura en Enfermería, Carrera de Enfermería, Facultad de Estudios Superiores Zaragoza, Universidad Autónoma de México. Correo electrónico: mtorres-x@, hotmail.com 


\section{Resumen}

Objetivo: Identificar la opinión de los jefes inmediatos sobre el desempeño de los egresados de la Facultad de Estudios Superiores Zaragoza para retroalimentar el plan de estudios y establecer un plan de mejoramiento continuo sobre las fortalezas y debilidades. Método: Estudio descriptivo, transversal y correlacional. Con una muestra no probabilística a conveniencia conformada por 29 jefes inmediatos en instituciones de salud en el segundo y tercer nivel de atención. Se aplicó el instrumento opinión del jefe inmediato sobre empleabilidad, satisfacción y desempeño laboral. El instrumento presentó una confiabilidad de 0,820 con la prueba de alfa de Cronbach y fue validado por expertos. Resultados: Para la contratación, el título se ubicó como la principal característica, seguido de la entrevista y el examen práctico. En relación con las características deseables del personal, se identificaron el trabajo en equipo y la búsqueda y manejo de información como las principales. Se observaron diferencias estadísticamente significativas entre las dimensiones características de contratación, satisfacción con el empleado y desempeño laboral y la institución de salud. Conclusiones: La evaluación del desempeño del egresado en el presente estudio fue la mejor preparación en la aplicación del proceso de enfermería, los conocimientos generales, la resolución de problemas, la toma de decisiones y la identificación institucional.

Palabras clave: estudiantes de enfermeria; evaluación del rendimiento de empleados; testimonio de experto; aptitud

\section{Immediate Supervisor Opinion about the Performance of Nursing Undergraduates}

\section{Abstract}

Aim: To identify the opinion of the immediate superiors about the performance of graduates of Facultad de Estudios Superiores Zaragoza for feedback on the curriculum and establish a continuous improvement plan on strengths and weaknesses. Methodology: Descriptive, cross-sectional and correlational study. With a nonrandom convenience sample comprised 29 immediate superiors in Health Institutions on 2nd and 3rd level of care. An instrument of supervisor on employability, job satisfaction and performance was applied. The instrument presented a 0.820 reliability with Cronbach Alpha test and was validated by experts. Results: For recruitment, the title is ranked as the main characteristic, followed by the interview and practice test. In relation to the desirable characteristics of staff teamwork, search and information management was identified as major. Statistically significant differences between the characteristic dimensions recruitment, employee satisfaction and job performance and health institution were observed. Conclusions: Performance evaluation of graduates in this study was the best training in the application of the nursing process, general knowledge, problem solving, decision-making and institutional identity.

Keywords: nursing students; employee performance appraisal; expert testimony; aptitude 


\section{Opinião do supervisor sobre o desempenho dos formandos do bacharelado em enfermagem}

\section{Resumo}

Objetivo: Identificar a opinião dos superiores imediatos sobre o desempenho dos formandos da Facultad de Estudios Superiores Zaragoza para o feedback sobre o currículo e estabelecer um plano de melhoria contínua nos pontos fortes e fracos. Método: Estudo descritivo, transversal e correlacionai. Com uma conveniência nonrandom amostra foi composta por 29 superiores imediatos nas instituições de saúde em $2^{\circ} \mathrm{e}$ $3^{\circ}$ nível de cuidados. O instrumento de avaliação do supervisor sobre a empregabilidade, a satisfação no trabalho e desempenho foi aplicado. O instrumento apresentou uma confiabilidade de 0,820 com o teste de Cronbach alfa e foi validado por especialistas. Resultados: Para o recrutamento, o título está classificado como a característica principal, seguido pela entrevista e teste. Em relação às características desejáveis de equipe trabalho em equipe, de pesquisa e informação de gestão foi identificada como major. Observaram-se diferenças estatisticamente significativas entre o recrutamento dimensões características, a satisfação dos funcionários e desempenho no trabalho e instituição de saúde. Conclusão: Avaliação do desempenho dos graduados neste estudo foi a melhor formação na aplicação do processo de enfermagem, conhecimentos gerais, resolução de problemas, tomada de decisão e participação institucional.

Palauras-chave: estudantes de enfermagem; avaliação de desempenho profissional; prova pericial; aptidão 


\section{Introducción}

En la actualidad, las universidades se encuentran sujetas a diversas presiones en función de los cambios vertiginosos respecto a las tendencias en la formación de los futuros profesionistas en las instituciones de educación superiores (IES) del mundo y en el ámbito nacional, producto de varios fenómenos como la globalización, la calidad que se le pide actualmente a los profesionistas (como una responsabilidad social a la población a la que sirven), la transición poblacional y epidemiológica, los avances en las tecnologias utilizadas en el sector de la salud para el cuidado de los usuarios de los servicios en los diferentes niveles de atención, la normatividad en el marco ético legal de la profesión, así como las situaciones particulares económicas, de salud y sociales del país. El contar con esta información les permite a las IES percibir las funciones profesionales para las que se preparó el estudiante - ahora profesionista- y cuáles de ellas responden a las necesidades sociales que las instituciones del sector de la salud requieren y valoran primordialmente (1).

Por otra parte, evaluar el desempeño profesional de los egresados permite medir su calidad en el ámbito laboral, recopilar información actualizada de los egresados y utilizar los resultados de un programa de formación en el área de la enfermería (2). En términos generales, se acepta que los estudios de graduados son importantes para aquellas instituciones preocupadas por la calidad de sus planes de estudio; además, el seguimiento de graduados permite conocer las características del empleo y del empleador, ya que estos últimos son quienes van a determinar las actividades para las que son contratados los profesionales y sus niveles de desempeño (3).

No existe una relación lineal entre lo que requiere el sector de salud público y el privado en función del tipo de atención que pretende satisfacer, su misión y visión, así como las políticas prioritarias en que se sustentan para responder a las necesidades sociales, lo que se proyecta en las características laborales que imperan. Sin embargo, aun cuando a veces es contradictorio el discurso del sector de la salud y sus programas prioritarios y las acciones que se realizan, las politicas y los recursos financieros que las respaldan, permite un acercamiento de información que retroalimente los planes de estudios de la carrera.

En la actualidad, la formación de un profesionista tiene tres aspectos básicos: a) el desarrollo del conocimiento general, b) el desarrollo del conocimiento laboral y c) la experiencia en el trabajo. Y de ahí que haya sido una tarea difícil para las IES integrar la teoría y la práctica en el contexto social, cultural y productivo, en el cual el futuro profesionista se desempeñará (4). Por otra parte, los empleadores reclutan egresados universitarios por cuatro razones: 1) están formados en las competencias que requieren las instituciones o empresas, 2) disponen de un conjunto de competencias transversales básicas que facilitan su incorporación a sectores en expansión, 3) se reduce el costo de inserción y adaptación y 4) constituyen una reserva de competencias que pueden ser movilizadas (5), motivos por los cuales el subsector de egresados universitarios está asociado directamente a la productividad de un país, ya 
que a través de las competencias desarrolladas por este grupo son capaces de adaptarse más fácilmente a los cambios que experimentan en economías globales y las demandas (6).

En el caso del área de la salud las competencias o perfiles adquiridos se relacionan y deben de estar cimentadas prioritariamente con la calidad de atención que requiere la población y solo a través de la investigación evaluativa se puede establecer el grado en que se logran los perfiles de egreso de los alumnos que se forman y así retroalimentar los programas de estudio y la formación de los docentes e infraestructura que se conjugan para ello, a través de las aportaciones de las instituciones de salud.

La formación del recurso humano, en el caso particular de enfermería, debe favorecer la articulación entre los conocimientos, las habilidades, las aptitudes y las actitudes conducentes a un desempeño adecuado y oportuno en diferentes contextos, donde la flexibilidad y la capacidad son claves para que los profesionales realicen su práctica social con saber, hacer y ser (7), y para lo cual fueron preparados. El desarrollo de estos atributos se corresponde con la formación en el ser (actitudes), en el pensar (aptitudes intelectuales), en el saber (conocimientos), en el hacer (habilidades) y en el aprendizaje logrado por medio de estas cuatro dimensiones da lugar a los llamados aprendizajes significativos, en los cuales el sujeto en formación reconfigura la información nueva con la experiencia, hasta integrar grandes cuerpos de conocimiento con sentido (8).

Para ello, el perfil profesional de la Carrera de Enfermería de la Facultad de Estudios Superiores Zaragoza de la Universidad Autónoma de México se basa en las funciones profesionales para la resolución de problemas desde un enfoque holístico. Dadas las anteriores consideraciones, el profesional de enfermería estará en capacidad de desempeñar las siguientes funciones profesionales (9):

Funciones asistenciales: proporcionar cuidados de enfermería con enfoque holístico y personalizado, aplicando teorías, modelos y el método propio de la disciplina que favorezca en el ser humano la solución de problemas de salud, inherentes al campo profesional y derivadas de las necesidades físicas, emocionales y sociales, para lograr el autocuidado y la adaptación funcional del hombre en el proceso salud-enfermedad, que se traduzca en bienestar y calidad de vida. Participa con el equipo multidisciplinario en el cuidado de la población, en los diferentes ámbitos de prevención, con base en el perfil epidemiológico del país.

Funciones educativas: participar en la planeación y desarrollo de programas de educación para la salud en la búsqueda de cambios de conducta y actitudes ante el proceso salud-enfermedad del individuo y su entorno.

Participar en la formación, actualización y desarrollo profesional de recursos humanos de enfermería de nivel técnico y licenciatura, mediante la actualización de conocimientos científicos y tecnológicos relacionados con la salud y la disciplina.

Funciones administrativas: aplicar la teoria, los métodos y las técnicas administrativas en los diferentes ámbitos de su intervención para garantizar 
la calidad de atención de enfermería al individuo, su familia y la comunidad, optimizando los recursos. Planear, organizar, dirigir y evaluar los servicios de enfermería en instituciones asistenciales, educativas e industriales, asî como en la práctica independiente.

Funciones de investigación: participar en la ejecución de proyectos de investigación epidemiológica, clínica y educativa, en instituciones de salud y servicios de enfermería, con enfermeras de grado y profesionales afines para la detección y posible solución de problemas de salud, así como para indagar y desarrollar sobre la práctica profesional vigente.

Con base en lo anterior, el estudio sobre el desempeño de los egresados se ha convertido en uno de los elementos para tomar en cuenta la congruencia externa de los planes de estudio y establecer directrices entre el mundo del empleo y el de la formación de una profesión. Por ello, el objetivo de esta investigación fue identificar la opinión de los jefes inmediatos sobre el desempeño de los egresados de la Facultad de Estudios Superiores Zaragoza para retroalimentar el plan de estudios y establecer un plan de mejoramiento continuo sobre las fortalezas y debilidades.

\section{Método}

El estudio fue de tipo descriptivo, transversal y correlacional, con una muestra no probabilística a conveniencia de 29 jefes inmediatos de 5 instituciones de salud en el segundo y tercer nivel de atención, ubicadas en la Ciudad de México. Se identificaron los informantes clave entre quienes estuvieran en el momento de la encuesta como dirigentes de enfermería, o mandos medios con responsabilidad en la contratación o supervisión de personal de enfermería y con participación en la toma de decisiones para la contratación de personal, ascensos o cambios de categoría y que tuvieran a su cargo egresados de la Facultad y que aceptaran contestar el cuestionario. La aplicación fue de autoadministración para facilitar la libertad de contestación de los empleadores.

\section{Instrumento}

Se aplicó el instrumento opinión al jefe inmediato sobre empleabilidad, satisfacción y desempeño laboral de los egresados. Para su elaboración se tomaron en cuenta las funciones profesionales del egresado, plasmadas en el plan de estudios de la carrera, y para cada una de estas se elaboraron diferentes ítems en los 5 apartados del instrumento. En el primer apartado se incluyen las variables sociodemográficas, como sexo y edad del jefe inmediato, tipo de unidad de adscripción y cargo que ocupan. La segunda parte aborda la dimensión "criterios de contratación": contar con un título de licenciatura, entrevista, examen psicométrico, examen de selección, experiencia previa. Los ítems cuentan con opciones de respuesta tipo Likert (1: muy importante; 2: importante, y 3: nada importante).

El tercer apartado se conforma por las características deseables del empleado: sexo, estado civil del aspirante, trabajo en equipo, búsqueda y 
manejo de información, toma de decisiones, buena presentación, capacidad de comunicación oral y escrita, capacidad de liderazgo, conocimientos de cómputo y manejo de un segundo idioma. Los ítems cuentan con opciones de respuesta tipo Likert (1: alta; 2: media, y 3: baja).

La dimensión de satisfacción con el empleado conforma el cuarto apartado, el cual considera las variables: conocimientos generales, conocimientos especializados, habilidad en la toma de decisiones, resolución de problemas, asumir responsabilidades, conocimientos de cómputo, trabajo en equipo, manejo ético, aplicación del Proceso Enfermero (PE) e identificación institucional. Los ítems cuentan con opciones de respuesta tipo Likert (1: totalmente satisfecho; 2: satisfecho; 3 : poco satisfecho, y 4: nada satisfecho).

La quinta sección aborda la dimensión sobre el desempeño laboral y las variables: puntualidad, asistencia, responsabilidad, interés y disposición, optimización de material, respeto por los pacientes, respeto por los compañeros, interés en su educación continua, respeto por la política institucional, participación con el equipo multiprofesional, participa en proyectos de investigación, aplica cuidados holísticos, aplica métodos disciplinares. Los 36 items cuentan con opciones de respuesta tipo Likert (1: siempre; 2: casi siempre; 3: a veces; 4: casi nunca, y 5: nunca).

El instrumento presentó una confiabilidad de 0,820 con la prueba alfa de Cronbach, el cual se sometió a validación de contenido por tres expertos en gestión y manejo de recursos hospitalarios.

En el presente estudio se cuidaron los aspectos éticos en relación con el consentimiento informado, al igual que la privacidad de los informantes y de las instituciones, señalados en el Reglamento de la Ley General de Salud en materia de investigación de México (10).

Para el procesamiento de los datos se utilizó el paquete de análisis estadístico (SPSS) versión 22, con el cual se realizó análisis descriptivo para las variables sociodemográficas, análisis de correlación y la prueba de KruskalWallis para las dimensiones señaladas en el instrumento.

\section{Resultados}

La muestra estuvo conformada por 29 jefes inmediatos, de quienes el $100 \%$ corresponde al sexo femenino, con una edad media de 42 $\pm 5,6$ años. De ellas, 22 corresponden a unidades de alta especialidad, y 7, a hospitales generales. Con relación al cargo que desempeñan, se contó con 2 enfermeras generales, una especialista, 20 jefas de servicio y 6 subjefas de enfermería.

En cuanto a los criterios de contratación, el contar con un título se ubicó como la principal característica, donde el $75 \%$ lo consideró muy importante; en segundo lugar, tanto la entrevista como el examen práctico fueron considerados por el $62 \%$ de los encuestados aspectos muy importantes; en tercer lugar, el examen psicométrico fue calificado por el 59\%, con la puntuación de mayor importancia; finalmente, la experiencia previa fue calificada por el 35\% como nada importante, y el 31\% lo calificó como muy importante (figura 1). 


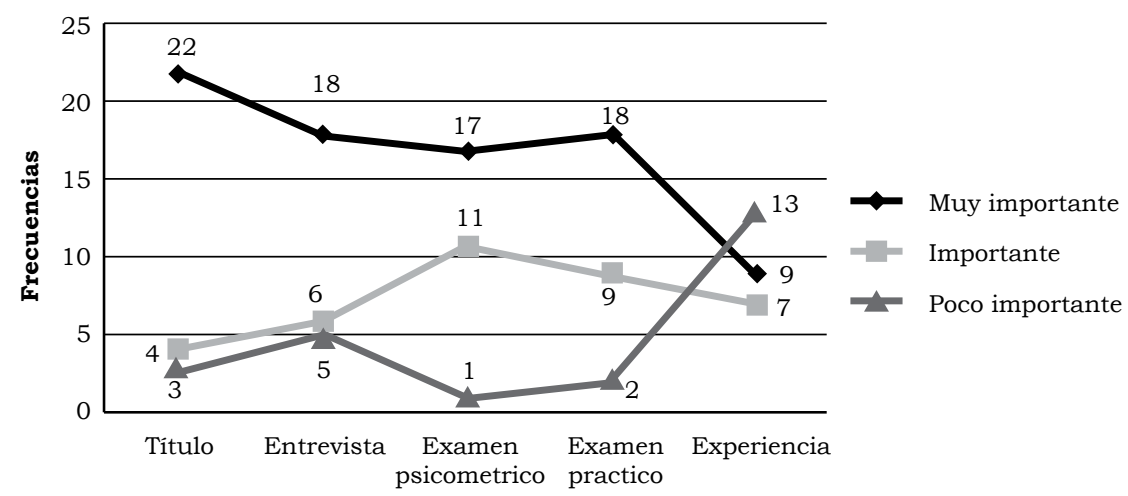

Figura 1. Criterios de contratación de personal

Fuente: elaboración propia, 2014

Con relación a las características deseables del personal, se identificaron como altamente deseables por 27 (93\%) jefes inmediatos el trabajo en equipo, la búsqueda y manejo de información y la toma de decisiones como los principales. En cuanto a la presentación y la capacidad de comunicación oral y escrita fueron 26 (90\%); entre tanto, la capacidad de liderazgo fue valorada con la máxima puntuación por 25 (86\%) de las personas encuestadas.

En cuanto al conocimiento de cómputo, el 65\% lo calificó como altamente deseable y el $27 \%$ lo consideró una característica medianamente deseable. El manejo de un segundo idioma fue tomado en cuenta solo por 11 jefes $(37 \%)$ con la máxima calificación; mientras que para $13(44 \%)$ es una característica medianamente deseable. Por último, el sexo y el estado civil del personal empleado es considerado por 15 (52\%) de los jefes inmediatos como una característica de baja importancia; mientras que el 34\% lo considera medianamente importante.

En las variables de satisfacción con el empleado, los jefes inmediatos presentaron las siguientes respuestas: siendo la aplicación del PE el mejor evaluado, con $52 \%$, que lo calificaron como totalmente satisfecho, y un $28 \%$, como satisfecho. La variable conocimientos generales se presenta en segundo lugar, ya que el $31 \%$ evaluó totalmente satisfecho, y el 52\%, como satisfecho. En cuanto opino que el empleado: el $65 \%$ resuelve problemas, el $62 \%$ toma de decisiones, el $58 \%$ tiene identificación institucional y el 55\% sabe trabajar en equipo, por lo que están satisfechos con el desempeño del personal de enfermería (figura 2).

En el caso de la satisfacción de como asume responsabilidades la enfermera/o a cargo, 11 (38\%) de las jefes inmediatas refieren estar altamente satisfechas. De igual manera, $11(38 \%)$ refieren estar satisfechas; mientras que $6(21 \%)$ mencionaron estar poco satisfechas. Con respecto a conocimientos en cómputo 10 (35\%) se encuentran totalmente satisfechas, $13(44 \%)$ satisfechas y $6(21 \%)$ poco satisfechas. En el caso de la variable sobre el manejo ético 7 (24\%) están totalmente satisfechas y 11 (38\%) se encuentran satisfechas; sin embargo, el $31 \%$ refiere estar poco satisfecha y el $7 \%$ nada satisfecha (figura 2). 
C. Generales

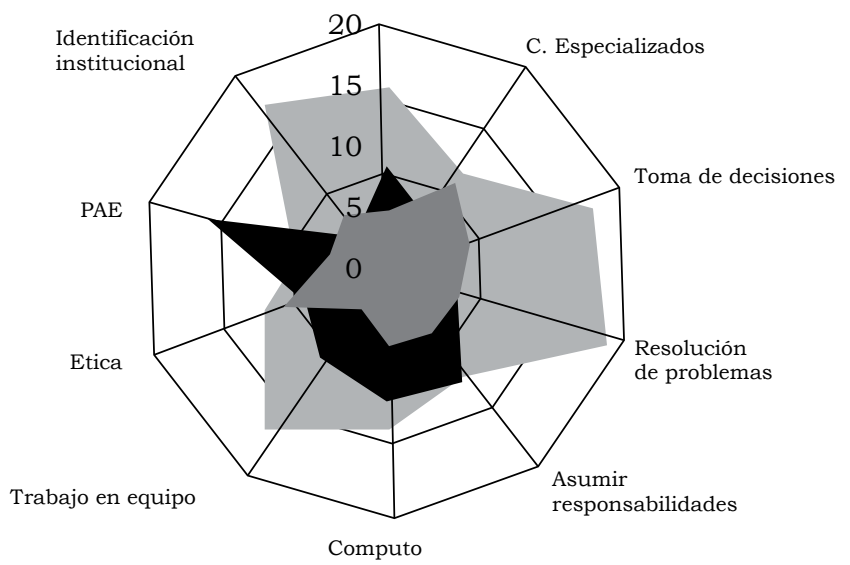

Satisfecho

Totalmente

Poco

Nada

Figura 2. Satisfacción con el empleado por variable

FUENTE: elaboración propia, 2014

Finalmente, la variable conocimientos especializados fue evaluada por 6 $(20 \%)$ de los jefes inmediatos como totalmente satisfecho, el 38\% lo calificaron como satisfechos; mientras que $9(31 \%)$ de ellos se encuentran poco satisfechos. Solo uno de ellos refiere estar nada satisfecho en este rubro y cabe señalar que $2(7 \%)$ mencionaron que no es una característica importante (figura 2).

En cuanto a la dimensión desempeño laboral, las variables con mejor evaluación por parte de los jefes inmediatos fueron el respeto por el paciente y por los compañeros de trabajo, donde el $97 \%$ (28) menciona que siempre se cumple; en segundo lugar, en las variables asistencia y responsabilidad, el $93 \%$ (27) de los encuestados lo refiere como siempre.

Las variables interés y disposición, colaboración con compañeros, respeto de la politicas institucionales, proporciona cuidados holisticos y aplicación de métodos disciplinares se posicionan en el tercer lugar, dado que el $86 \%$ (25) refirió que siempre se lleva a cabo.

Los aspectos con menor evaluación fueron el interés en educación continua, donde el $68 \%$ (20) de los jefes respondió que siempre se realiza; el $28 \%$ (8) menciona que solo en algunas ocasiones, y uno contestó que nunca se lleva a cabo. La participación en proyectos de investigación continúa siendo un punto que resalta, ya que en este caso el $41 \%$ (12) de los encuestados respondió que nunca se participa, el 34\% (10) contestó que algunas veces lo hacen y el 7 (24\%) de ellos afirma que siempre se realiza.

De acuerdo con el análisis de correlación, se observa que el desempeño laboral y la satisfacción con el empleado presenta una correlación fuerte con un valor de $p=0,000$. En cuanto a las características de contratación, presenta una correlación negativa media, tanto con la satisfacción con el empleado como con el desempeño laboral. El resto de las dimensiones analizadas no presenta significancia estadística entre las correlaciones observadas (tabla 1$)$. 
TABLA 1. Correlaciones por dimensiones

\begin{tabular}{|l|c|c|c|}
\hline \multicolumn{1}{|c|}{ Variables } & $\begin{array}{c}\text { Caracteristicas deseables } \\
\text { del empleado }\end{array}$ & $\begin{array}{c}\text { Satisfacción con } \\
\text { el empleado }\end{array}$ & $\begin{array}{c}\text { Desempeño } \\
\text { laboral }\end{array}$ \\
\hline $\begin{array}{l}\text { Características } \\
\text { de contratación }\end{array}$ & NS & $-0,551^{*}$ & $-0,516^{*}$ \\
\hline $\begin{array}{l}\text { Características } \\
\text { deseables del } \\
\text { empleado }\end{array}$ & \multirow{2}{*}{\begin{tabular}{c} 
NS \\
\hline $\begin{array}{l}\text { Satisfacción con } \\
\text { el empleado }\end{array}$
\end{tabular}} & $\ldots, 002$ & 0,004 \\
\hline
\end{tabular}

*Prueba de correlación de Pearson. La correlación es significativa en el nivel 0,01 (2 colas).

Fuente: elaboración propia

Se observaron diferencias estadísticamente significativas entre las dimensiones caracteristicas de contratación, satisfacción con empleado y desempeño laboral y la institución de salud; sin embargo, las características deseables del empleado no presentan diferencias por la misma variable (tabla 2). En cuanto a la variable cargo que ostenta el jefe inmediato, al compararse por las mismas dimensiones, ninguna de ellas presenta diferencias estadísticas. Por ello se puede observar que no existen diferencias con respecto al cargo; pero sí a la institución de salud.

TABLA 2. Rangos por dimensión e institución de salud

\begin{tabular}{|c|c|c|c|c|}
\hline Variables & Institución & $\mathbf{n}$ & Rango promedio & $\mathbf{p}$ \\
\hline \multirow{5}{*}{$\begin{array}{l}\text { Características } \\
\text { de contratación }\end{array}$} & INCMNSZ & 5 & 6,70 & \\
\hline & INP & 9 & 21,72 & \\
\hline & HRAEI & 5 & 8,30 & $0,000 *$ \\
\hline & HJM & 3 & 10,83 & \\
\hline & IMMS & 7 & 18,86 & \\
\hline \multirow{5}{*}{$\begin{array}{l}\text { Características } \\
\text { deseables } \\
\text { del empleado }\end{array}$} & INCMNSZ & 5 & 16,60 & \\
\hline & INP & 9 & 18,61 & \\
\hline & HRAEI & 5 & 10,70 & NS \\
\hline & HJM & 3 & 18,50 & \\
\hline & IMMS & 7 & 10,79 & \\
\hline \multirow{5}{*}{$\begin{array}{l}\text { Satisfacción con el } \\
\text { empleado }\end{array}$} & INCMNSZ & 5 & 26,00 & \\
\hline & INP & 9 & 9,61 & \\
\hline & HRAEI & 5 & 12,00 & $0,003^{*}$ \\
\hline & HJM & 3 & 19,50 & \\
\hline & IMMS & 7 & 14,29 & \\
\hline \multirow{5}{*}{ Desempeño laboral } & INCMNSZ & 5 & 24,10 & \\
\hline & INP & 9 & 9,50 & \\
\hline & HRAEI & 5 & 18,30 & $0,021^{*}$ \\
\hline & HJM & 3 & 14,83 & \\
\hline & IMMS & 7 & 13,29 & \\
\hline
\end{tabular}

*Prueba de Kruskal-Wallis, con valor de significancia $<0,05$.

Fuente: elaboración propia 


\section{Discusión}

La evaluación del comportamiento de los egresados conforme a valores inherentes a calidad en la atención como profesionales con responsabilidad y dedicación, orientación hacia el paciente, respetuosos y con compromiso ético, son una muestra positiva de aspectos que deben permanecer como distintivos de la actitud que caracterice al profesional de enfermeria y que han sido identificados en otros estudios de evaluación del desempeño (11). Esto coincide con nuestro estudio, ya que en la dimensión de desempeño laboral las variables con mejor evaluación por parte de los jefes inmediatos fueron el respeto por el paciente y por los compañeros de trabajo, así como la asistencia y responsabilidad.

La evaluación realizada por los jefes inmediatos destaca como las características del egresado más deseables, en orden de importancia, las siguientes: el trabajo en equipo, la búsqueda y manejo de información, la toma de decisiones, la comunicación oral y escrita, la buena presentación y la capacidad de liderazgo. En prioridad intermedia, el conocimiento de cómputo y el manejo de un segundo idioma. Lo anterior concuerda, en varios elementos, con el estudio de Muñoz y cols. (7), donde se identifican como los aspectos más importantes para los jefes: la comunicación por escrito; el trabajo bajo presión; el identificar, planear y resolver; la formación en valores y principios, y el trabajo en equipo. Mientras que, de la misma manera, se le da una mediana importancia a las herramientas informáticas y al manejo de otro idioma. Lo anterior antepone un conflicto, ya que, por un lado, se les pide tanto a las IES como al sector de la salud su incorporación en procesos de evaluación, ya sea acreditación o certificación, internacionales y, al mismo tiempo, son los cargos directivos propios de las instituciones empleadoras los que minimizan la importancia de que el personal tenga un dominio de estas características.

En cuanto a la evaluación del desempeño del egresado, en el presente estudio se identificaron de mejor preparación la aplicación del $\mathrm{PE}$, los conocimientos generales, la resolución de problemas, la toma de decisiones y la identificación institucional; mientras que en cómo asume responsabilidades, el egresado fue evaluado en un punto intermedio y en el caso del manejo ético existe una tercera parte de los jefes inmediatos que no se encuentran satisfechos con esta característica. En el estudio realizado por Torres, Sierra y Pinzón (2) se identificó una situación similar en el componente ético, donde ellos reportan que el $65 \%$ fue evaluado como excelente; sin embargo, existe un porcentaje considerable que obtuvo una evaluación inferior. Esta situación antepone un punto que debe ser analizado y de reflexión sobre las tres partes involucradas: que se aborda en las IES curricularmente sobre este aspecto, como lo aplica el personal de salud en su desempeño laboral y que considera el jefe inmediato como un comportamiento éticamente adecuado. En cuanto al resto de los elementos en el mismo estudio, así como en el realizado por González y Arras (12), donde se identificó con la mayor satisfacción el fundamento científico en el cuidado de enfermería y en segundo lugar el uso del $\mathrm{PE}$, utiliza recursos de manera eficiente y realiza un trabajo propositivo, aspectos observados de manera similar en el presente estudio. 
Mientras que otros aspectos como la asistencia, la puntualidad, la colaboración con compañeros y el respeto por las politicas institucionales también obtuvieron valoración altas por parte de los jefes, lo cual coincide con el estudio de Daberkow y Sánchez (13), donde se establece que los empleadores están de acuerdo con la formación y los conocimientos proporcionados por la facultad a sus egresados y con el estudio de García y López (14), donde se encontró que los egresados se caracterizan, además, por un desempeño acorde con las características de la institución, participan en procesos de mejoramiento continuo para la prestación de servicios y ponen en práctica las políticas públicas en salud; así mismo, poseen y se esfuerzan por adquirir los conocimientos requeridos de la normatividad y legislación en salud relacionados con su área de trabajo.

Se presentaron dos aspectos evaluados con puntajes bajos en nuestros egresados por parte de los jefes: el interés por la educación continua y la participación en investigación. Este último aspecto contrasta con el del estudio de González y Arras (12), en el cual reportan que cerca del 60\% participa en ensayos clínicos dentro de las instituciones, aunque se no refiere qué tipo de participación llevan a cabo.

Lo anterior nos lleva a querer seguir trabajando con los egresados, ya que sin duda la participación de enfermeria en investigación, es un elemento que necesita ser reforzado, considerando que es una función profesional que está plasmada en el plan de estudios y es necesario no solo trabajarlo por las IES, sino por las instituciones de salud, donde se debe promover y fomentar la participación en todos los niveles de atención.

\section{Conclusiones}

El mercado de trabajo está enmarcado por dos grandes tendencias: la globalización y una economía basada en el conocimiento. La subcontratación, la rapidez para incorporar nuevos cambios, la innovación tecnológica, las nuevas formas de distribución, la búsqueda continua de la eficiencia, la alta movilidad del recurso humano, la flexibilidad organizacional, el uso intensivo de las tecnologías de la información y la comunicación (TIC) generan nuevas formas de producción y mercados de alto valor agregado donde el capital humano tiene un papel central (15).

Para el jefe inmediato, las características más deseables de los egresados fueron el trabajo en equipo, la búsqueda y manejo de información, la toma de decisiones, la comunicación oral y escrita, la buena presentación y la capacidad de liderazgo.

Otro aspecto importante en la evaluación del desempeño del egresado en el presente estudio fue la mejor preparación en la aplicación del proceso de enfermería, los conocimientos generales, la resolución de problemas, la toma de decisiones y la identificación institucional.

Por último, es necesario destacar que los egresados, de acuerdo con la opinión de los jefes inmediatos, cumplen las funciones profesionales para las cuales fueron preparados a lo largo de los cuatro años que dura la carrera. 


\section{Financiamiento}

No se solicitó apoyo financiero para este estudio.

\section{Conflicto de interés}

Los autores declaran no tener ningún conflicto de interés.

\section{Referencias}

1. Estrella VG, Ponce LM. Impacto laboral de egresados universitarios y opinión de empleadores. México: Universidad Autónoma de Baja California: Miguel Ángel Porrúa; 2006.

2. Torres CC, Sierra MK, Pinzón SJ. Evaluación del desempeño de los egresados del programa de enfermería de la Universidad Santander 2004-2010. Rev Cuid. 2012;3(3):270-9.

3. Escalona RL, Fernández CE. Los estudios de egresados del área bibliotecológica: de la teoría a la práctica. Rev Interam Bibliot. 2007;(30)2:113-35.

4. Argüelles A, Gonzci A. Educación y capacitación basada en normas de competencias: una perspectiva internacional. México: Limusa; 2001.

5. Salas DI, Murillo GF. Los profesionistas universitarios y el mercado laboral mexicano: convergencias y asimetrias. Revista de la Educación Superior. 2013;42(165):63-81.

6. Urciaga J, Almendárez M. Salarios, educación y sus rendimientos en la fron $\neg$ tera norte de México. Un estudio de capital humano. Región y Sociedad. 2008;20(41):33-56.

7. Muñoz MN, Rodríguez P, Hincapié J, Agudelo AM y Ramírez R. Percepción de empleadores sobre las competencias de graduados del programa de enfermería de la Fundación Universitaria del Área Andina de Pereira (Colombia). Cult Cuid Enferm. 2013;9(2):22-38.

8. Diaz BA, Hernández RG. Estrategias docentes para un aprendizaje significativo: una interpretación constructivista. $3^{a}$ ed. México: McGraw Hill; 2010.

9. Facultad de Estudios Superiores Zaragoza. Plan de Estudios de la Carrera de Enfermería. Facultad de Estudios Superiores Zaragoza. México: UNAM; 1997.

10. Reglamento de la Ley general de Salud en Materia de Investigación para la Salud. Última reforma publicada DOF 02-04-2014. México. [Citado: 22 de julio de 2015]. Disponible en: http://www.salud.gob. $\mathrm{mx} /$ unidades/cdi/nom/compi/rlgsmis.html

11. Torres Contreras CC, Sierra Mujica KS, Pinzón Saavedra JD. Evaluación del desempeño de los egresados del Programa de Enfermería de la Universidad de Santander 2004-2010. Revista CUIDARTE. 2012;3(3):270-9.

12. González CE, Arras VA. Profesionalización de la enfermería y desempeño, desde la óptica del jefe inmediato superior en Chihuahua, Chihuahua. European Scientific Journal. 2014;10(1):312-26. 
13. Daberkow HF, Sánchez EA, Fernández BF, Ruiz MM, Tenorio GM, Castro OM. Opinión de empleadores en las instituciones de salud sobre el desempeño laboral de egresados de la licenciatura en Enfermería de una Universidad pública en la ciudad de Veracruz, México. Revista Médica Electrónica Portales Médicos [internet]. 2013 [citado 2015 jul 28];8(3):[5 p]. Disponible en: http://www.revista-portalesmedicos.com/ revista-medica/empleadores-laboral-licenciatura-enfermeria/

14. García OC, López RL, Osorio GL, Realpe DC. Desempeño profesional de los egresados del programa de enfermería de la universidad de Caldas y su relación con la ley de seguridad social en salud y con el perfil de formación (Manizales 1995-2004). Hacia la Promoción de la Salud. 2007;12:91-108.

15. Diaz PC. Tendencias y requerimientos del mercado de trabajo en la economía del conocimiento: estudio sobre los egresados del Centro Universitario de Ciencias Económico Administrativas de la Universidad de Guadalajara. Revista de la Educación Superior. 2012;41(161):9-30. 